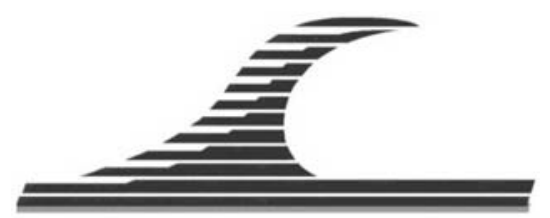

\title{
Etude de l'évolution des formes chimiques des métaux dans des sédiments marins dragués stockés à terre
}

\author{
Fabienne SEBY ${ }^{1}$, Christelle BENOIT-BONNEMASON ${ }^{1}$, Emmanuel TESSIER ${ }^{2}$, \\ Claude ALZIEU ${ }^{3}$, Jean-Luc AQUA ${ }^{3}$, Laurent SANNIER ${ }^{3}$, Olivier F.X. DONARD ${ }^{2}$ \\ ${ }^{1}$ UT2A, Hélioparc Pau-Pyrénées, 2 avenue Pdt Angot, 64053 Pau cedex 9, France. \\ fabienne.seby@univ-pau.fr \\ ${ }^{2}$ LCABIE / IPREM, UMR CNRS/UPPA 5254, Hélioparc Pau-Pyrénées, \\ 2 avenue Pdt Angot, 64053 Pau cedex 9, France. \\ ${ }^{3}$ Conseil Général du Var, Direction des Ports, 390 avenue des Lices, BP1303, \\ 83076 Toulon cedex, France.
}

\section{Résumé :}

Le stockage à terre des sédiments est un traitement qui a été envisagé dans le cadre du projet SEDI.MAR.D. 83 dans le but de diminuer la charge polluante des sédiments marins dragués. Ce traitement serait susceptible d'entraîner, par vieillissement naturel, des transformations physico-chimiques et/ou biologiques au sein de la matrice sédimentaire qui pourraient modifier sa toxicité. Ce travail a porté sur le devenir des métaux et de leurs formes chimiques lors du vieillissement des sédiments.

Ce traitement permet d'abaisser la moitié de la concentration initiale du tributylétain (TBT) après deux mois d'entreposage. Cette dégradation du TBT au cours du temps de stockage à terre s'accompagne de la formation d'espèces de l'étain qui sont moins dangereuses. Pour l'arsenic, seules les formes inorganiques As(III) et $\mathrm{As}(\mathrm{V})$ ont été détectées. As(III) est la forme majoritaire dans le sédiment fraîchement dragué et s'oxyde en $\mathrm{As}(\mathrm{V})$ en fonction de la durée du traitement. Cette oxydation a également été observée pour le chrome, avec apparition de très faibles quantités de chrome hexavalent. En raison de sa grande toxicité, la formation du $\operatorname{Cr}(\mathrm{VI})$ est une limite au traitement étudié. Il semble donc préférable de considérer un temps de stockage à terre assez court de façon à dégrader le TBT tout en minimisant la formation de Cr(VI). Dans la mesure où aucun traitement vraiment efficace n'existe pour gérer les sédiments marins contaminés, cette étude montre que le stockage à terre peut être considéré comme une première approche peu coûteuse. Si le degré de pollution est suffisamment abaissé par ce procédé, il est ensuite possible d'envisager une valorisation de ces matériaux dans le domaine des travaux publics par exemple (remblais).

Soumis le 26 février 2009, accepté le 2 septembre 2009, en ligne le 23 novembre 2009.

La seule version examinée est celle écrite en français. La ou les autres versions n'étant pas examinées par le comité de rédaction de la revue, sont donc publiées sous l'entière responsabilité du ou des auteurs.

\section{A TRANSLATED VERSION IN ENGLISH IS AVAILABLE ONLINE}

Pour citer cet article :

SEBY F., BENOIT-BONNEMASON C., TESSIER E., ALZIEU C., AQUA J.-L., SANNIER L., DONARD O.F.X. (2009). Etude de l'évolution des formes chimiques des métaux dans des sédiments marins dragués stockés à terre. Revue Paralia, $\mathrm{n}^{\circ} 2$, pp s3.1-s3.12.

DOI: 10.5150/revue-paralia.2009.s03 ～(disponible en ligne - http:/www.paralia.fr - available online) 


\section{Introduction}

Le projet SEDI.MAR.D. 83 a été initié par le Conseil Général du Var (France) afin d'établir et d'évaluer différentes procédures de gestion à terre de sédiments marins dragués. Parmi les différents traitements étudiés, le stockage à terre a été considéré car ce traitement pourrait permettre une diminution de la toxicité des sédiments par atténuation naturelle. En effet, le vieillissement du sédiment peut engendrer des transformations chimiques, physiques et/ou biologiques qui peuvent modifier la structure de certaines molécules toxiques pour les transformer en des composés moins dangereux selon un processus très naturel puisqu'aucun additif n'est introduit dans le sédiment. Ce procédé a déjà montré son efficacité pour les molécules organiques telles que les hydrocarbures aromatiques polycycliques (AMIR et al., 2005 ; VERMEULEN et al., 2007) mais aucune étude n’a encore été réalisée pour comprendre le devenir des formes chimiques des métaux lors du processus de vieillissement à terre des sédiments marins.

La charge en métaux étant importante dans les sédiments méditerranéens, le comportement de certains métaux et de leurs formes chimiques a été étudié dans des sédiments dragués qui ont été stockés à terre pendant 10 mois. Dans la plupart des études, seules les concentrations en métaux totaux sont prises en compte et il est généralement considéré que la mobilité des métaux augmente avec le vieillissement (CAILLE et al., 2003 ; CAPPUYNS et al., 2006). Les métaux sont généralement libérés par l'oxydation des sédiments prélevés en anoxie (CAILLE et al., 2003 ; CAPPUYNS et al., 2006). Ce type de comportement a été mis en évidence pour des sédiments de rivière mais, à notre connaissance, ce comportement reste encore assez méconnu dans le cas de sédiments marins qui présentent des caractéristiques physico-chimiques différentes.

Par ailleurs, les transformations physico-chimiques et/ou biologiques liées au vieillissement du sédiment peuvent engendrer une modification des formes chimiques des métaux lors de ce traitement. Les analyses de spéciation qui permettent d'accéder à cette information sont intéressantes dans ce contexte car elles vont permettre de bien caractériser le sédiment dragué mais aussi de mieux comprendre l’impact environnemental associé au stockage à terre des sédiments. Par exemple, il est bien connu que l'étain minéral est inoffensif mais les composés organiques de l'étain comme les butylétains sont dangereux et la toxicité augmente avec le nombre de groupement butyle (CRAIG, 1986). Il est important de caractériser le TBT et ses produits de dégradation comme le dibutylétain (DBT) et le monobutylétain (MBT) dans les sédiments car le TBT a été utilisé durant les deux dernières décennies en tant qu'agent antisalissure dans les peintures pour bateaux et subsiste dans la masse sédimentaire (ALZIEU, 2000). Le mercure est généralement présent sous forme inorganique mais peut être fortement affecté par des processus de méthylation/déméthylation qui vont affecter son devenir et sa toxicité. La méthylation est un processus biologique qui 
transforme le mercure inorganique en méthylmercure qui est facilement bioaccumulé et sa présence est donc amplifiée le long de la chaîne alimentaire (CRAIG, 1986). La connaissance de l'évolution des espèces arséniées pendant ce traitement est également importante car le stockage à terre peut transformer les formes inorganiques toxiques (As(III) et $\mathrm{As}(\mathrm{V})$ ) en formes organiques qui le sont moins (e.g. MMA, DMA, AsB) (O’NEILL, 1995). Dans les sédiments, le chrome peut exister sous forme de chrome trivalent (Cr(III)) et de chrome hexavalent (Cr(VI)). Si le $\mathrm{Cr}(\mathrm{III})$ est essentiel pour de nombreux organismes vivants, le $\mathrm{Cr}(\mathrm{VI})$ est classé comme composé carcinogène (KRETCHIK, 2005). Seules des analyses de spéciation peuvent donner une information au niveau de la toxicité liée au chrome dans les sédiments.

Le suivi des formes chimiques de quelques éléments d'importance dans les sédiments marins dragués disposés à terre sur une longue période semble donc être une bonne approche pour mieux estimer l'impact et la toxicité des métaux dans la masse sédimentaire. Aussi, le but de ce travail est de mieux connaître la transformation des espèces métalliques dans les sédiments marins dragués en fonction de leur vieillissement lors d'un procédé de traitement par stockage à terre dans des conditions environnementales réelles.

\section{Matériel et méthodes}

\subsection{Le stockage à terre des sédiments}

Les traitements appliqués aux sédiments sur le site pilote mis en place dans le cadre du projet SEDI.MAR.D. 83 sont résumés figure 1.

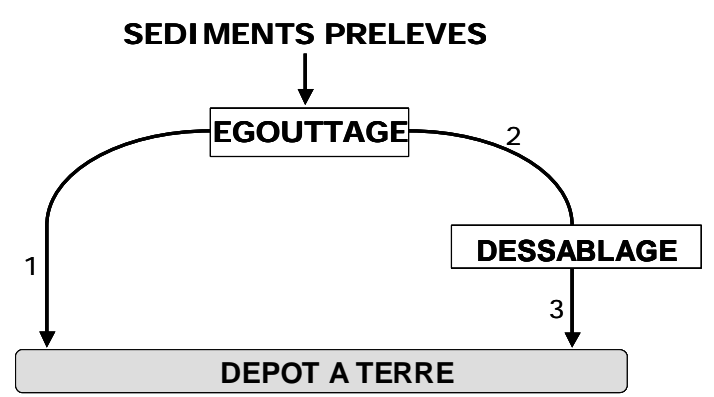

Figure 1. Traitements in situ appliqués aux sédiments.

Après dragage de différents ports de la région Provence Alpes Côte d'Azur (PACA) (France), les sédiments prélevés ont été transportés sur le site pilote de SEDI.MAR.D 83 situé à la Seyne-sur-Mer (France). Ils ont tout d'abord été stockés sur une plateforme étanche et les eaux de lixiviation ont été collectées et traitées avant d'être remises dans le milieu naturel. Après cette phase de drainage, une partie des sédiments a été stockée à terre sur la plateforme étanche sans traitement supplémentaire (figure 1, chemin 1). 
Cette fraction est appelée sédiments bruts dans la suite de ce travail. Le sable a été éliminé dans l'autre partie des sédiments (figure 1, chemin 2) pour séparer la fraction fine où sont concentrés les composés polluants de la fraction sableuse qui est généralement moins contaminée. Suite à cette procédure, les sédiments ont été stockés à terre (figure 1, chemin 3). Les sédiments ont été stockés sur la plateforme pendant 10 mois et ils ont été humidifiés et retournés mécaniquement une fois par semaine pour favoriser leur oxygénation. Des prélèvements ont été effectués tous les deux mois pendant dix mois afin de pouvoir suivre les métaux ainsi que les formes chimiques de certains d'entre eux. Un prélèvement sous atmosphère inerte a été impossible à réaliser pour des raisons techniques. Pour bloquer les cinétiques d'évolution, les sédiments ont été immédiatement congelés à $-18^{\circ} \mathrm{C}$ après leur prélèvement.

\subsection{Procédure analytique}

Les paramètres mesurés dans les sédiments dragués (bruts et dessablés) sont les concentrations en $\mathrm{Ni}$, As, Sb, Cd, Sn, Se, $\mathrm{Hg}, \mathrm{Pb}, \mathrm{Mn}, \mathrm{Zn}, \mathrm{Cr}, \mathrm{Cu}$, Fe et $\mathrm{Al}$ ainsi que les formes chimiques de As, Cr, Sn et Hg. La procédure analytique utilisée est présentée figure 2. Cette procédure a été associée à des procédures de contrôle qualité strictes, notamment par l'utilisation de matériaux de référence certifiés.

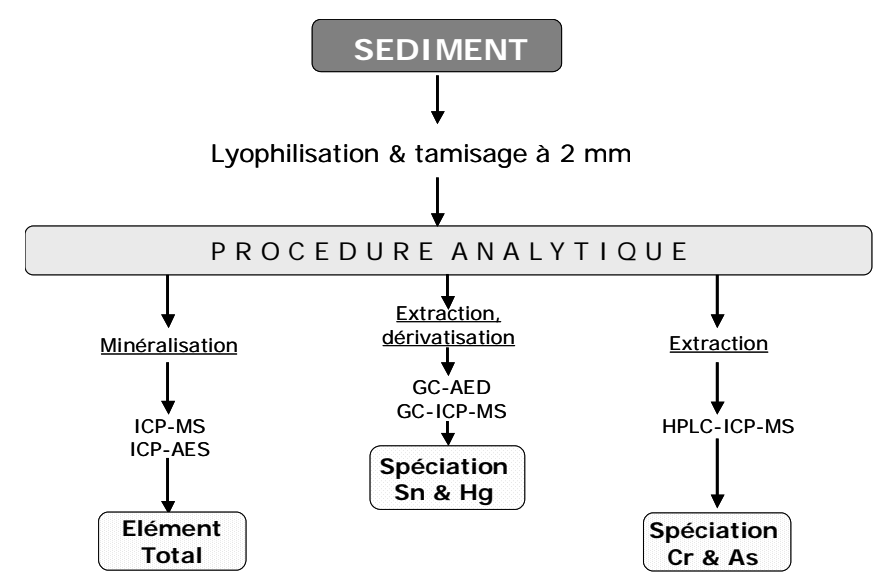

Figure 2. Procédure analytique appliquée aux sédiments.

Les détails techniques concernant ces analyses sont disponibles dans différents ouvrages (SEBY et al., 2007 ; DIRKX et al., 1994 ; RODRIGUEZ MARTIN-DOIMEADIOS et al., 2003 ; THOMAS et al., 1997 ; SEBY et al., 2003). Brièvement, l'analyse des métaux est réalisée par spectrométrie de masse à plasma induit (ICP-MS) (Ni, As, Sb, $\mathrm{Cd}, \mathrm{Sn}, \mathrm{Se}, \mathrm{Hg}, \mathrm{Pb}$ ) ou spectrométrie d'émission atomique à plasma induit (ICP-AES) $(\mathrm{Mn}, \mathrm{Zn}, \mathrm{Cr}, \mathrm{Cu}, \mathrm{Fe}$ et $\mathrm{Al}$ ) après minéralisation sous champs micro-ondes en milieu acide nitrique et acide fluorhydrique. Les formes chimiques des métaux ont été dosées à l'aide de méthodes couplées associant en ligne une séparation par chromatographie (phases liquide ou gazeuse) et une détection spectrométrique. Les composés 
organostanniques sont déterminés après extraction sous champs micro-ondes en milieu acide acétique. Afin de transformer les organoétains extraits en une forme volatile analysable par chromatographie en phase gazeuse, une étape de dérivatisation par éthylation est réalisée avant l'analyse par chromatographie en phase gazeusespectrométrie d'émission atomique (GC-AED). Les espèces du mercure sont analysées par couplage chromatographie en phase gazeuse-spectrométrie de masse à plasma induit (GC-ICP-MS) après extraction sous champs micro-ondes en milieu $\mathrm{HNO}_{3} 6 \mathrm{M}$ et éthylation.

La spéciation de l'arsenic et du chrome a été réalisée avec le couplage chromatographie en phase liquide-spectrométrie de masse à plasma induit (HPLC-ICP-MS). Dans les deux cas, le mécanisme de séparation utilisé est l'échange d'anions. Pour les espèces de l'arsenic, l'extraction est réalisée en milieu acide phosphorique 1M sous champs microondes. Dans le cas du chrome, l'extraction est menée en milieu basique (tampon $\mathrm{NH}_{4}{ }^{+} / \mathrm{NH}_{3} \mathrm{à} \mathrm{pH}$ 9) dans un bain ultrasonique. Un tel milieu ne permet d'extraire que le chrome hexavalent, le chrome trivalent restant sous une forme précipitée à ce pH.

\section{Résultats et Discussion}

\subsection{Evolution des concentrations en métaux en fonction du temps de stockage à terre}

Les teneurs de la plupart des éléments analysés dans les sédiments étudiés sont très souvent supérieures aux niveaux établis par GEODE. Ces niveaux ont été établis pour donner des références aux gestionnaires de ports pour aider à la décision du devenir des sédiments dragués. Deux seuils (N1 et N2) ont été définis pour caractériser la qualité d'un sédiment. N1 est le niveau au dessous duquel le clapage en mer est autorisé et N2 représente le seuil au dessus duquel le clapage en mer est interdit. Entre N1 et N2, une caractérisation complète du sédiment doit être réalisée. Dans le sédiment étudié dans ce travail, les concentrations en As, $\mathrm{Cd}, \mathrm{Hg}, \mathrm{Pb}, \mathrm{Zn}$ et $\mathrm{Cu}$ sont supérieures au niveau N2. Seuls ces éléments sont référencés par GEODE. Le clapage en mer est donc interdit et ce sédiment doit être considéré comme un déchet à terre dangereux.

Dans le cas des sédiments dessablés, les teneurs en métaux restent sensiblement constantes pendant les dix mois de stockage alors qu'une diminution des concentrations est observée pour les sédiments bruts avec obtention d'un plateau après quatre mois de vieillissement. La baisse des concentrations est importante pendant les deux premiers mois et diminuent ensuite de façon plus progressive. Compte tenu des incertitudes de mesure, il semble que pour l'ensemble des éléments étudiés, les concentrations se stabilisent entre le $4^{\text {ème }}$ et le $10^{\text {ème }}$ mois. Les concentrations en métaux restent toutefois élevées après 10 mois et sont supérieures au niveau N2. Pour les éléments majeurs tels que $\mathrm{Fe}, \mathrm{Mn}$ et $\mathrm{Al}$, deux comportements peuvent être différenciés. Alors que le fer et l'aluminium ont des teneurs qui diminuent selon les mêmes tendances que celles des éléments traces, les concentrations en manganèse diminuent assez peu pendant les deux 
premiers mois (15\% de baisse) et restent stables par la suite. L'évolution du carbone organique total suit la même tendance que celles du fer et de l'aluminium ; une baisse de $20 \%$ est observée les deux premiers mois et les valeurs sont stables ensuite.

D’après ces résultats, il semble qu'une période de stockage à terre de deux à quatre mois soit nécessaire pour oxyder les sédiments fraîchement dragués. Ce comportement est moins significatif pour les sédiments dessablés car le traitement de dessablage en luimême entraîne une oxydation rapide du sédiment et la concentration en métaux reste stable après le dépôt à terre.

Les concentrations en métaux plus importantes dans les sédiments dessablés par rapport aux sédiments bruts peuvent être expliquées par la faible affinité des métaux pour le sable. Seul l'arsenic présente des niveaux de concentration très proches dans les deux types de sédiments. Cet élément semble donc peu influencé par la présence de sable, ce qui laisse penser que cet élément a une affinité similaire avec tous les types de particules.

Il a déjà été montré que les changements redox qui affectent les métaux se produisent sur une échelle de temps inférieure à un an (SINGH et al., 1998). Le séchage et l'oxydation des sédiments initialement en anoxie sont effectifs pendant les deux premières semaines. Pendant cette période, les métaux initialement présents sous forme insoluble deviennent progressivement disponibles (STEPHENS et al., 2001). En effet, la distribution de certains éléments dans les sédiments anoxiques est généralement dominée par la formation de sulfures métalliques (CAILLE et al., 2003 ; CAPPUYNS et al., 2006). Le mercure et le cuivre peuvent aussi être présents en quantité appréciable dans la structure de la pyrite (CAILLE et al., 2003). De plus, de grandes quantités de matière organique s'accumulent dans les sédiments anoxiques car l'activité microbiologique est réduite dans les environnements pauvres en oxygène. Ces composés organiques naturels sont souvent considérés comme des réservoirs de polluants métalliques. En conséquence, il est maintenant reconnu que l’oxydation du matériel dragué peut conduire à une modification de la mobilité des métaux (CAILLE et al., 2003 ; CAPPUYNS et al., 2006 ; STEPHENS et al., 2001). Il a cependant été montré que la solubilité des métaux augmente rapidement pendant les premières étapes de l'aération mais une rapide ré-adsorption des métaux se produit sur les nouvelles phases porteuses qui se forment après l'étape d'oxydation (CAILLE et al., 2003).

3.2 Evolution des organoétains dans les sédiments en fonction du temps de dépôt à terre Les concentrations en butylétains pendant les 10 mois de dépôt à terre des sédiments bruts sont présentées figure 3. Un profil similaire a été obtenu pour les sédiments dessablés.

Les teneurs en butylétain dosées dans les sédiments sont élevées avec une concentration en TBT 20 fois supérieure au niveau N2 recommandé par GEODE. Ces valeurs empêchent totalement le clapage en mer de ces sédiments. 


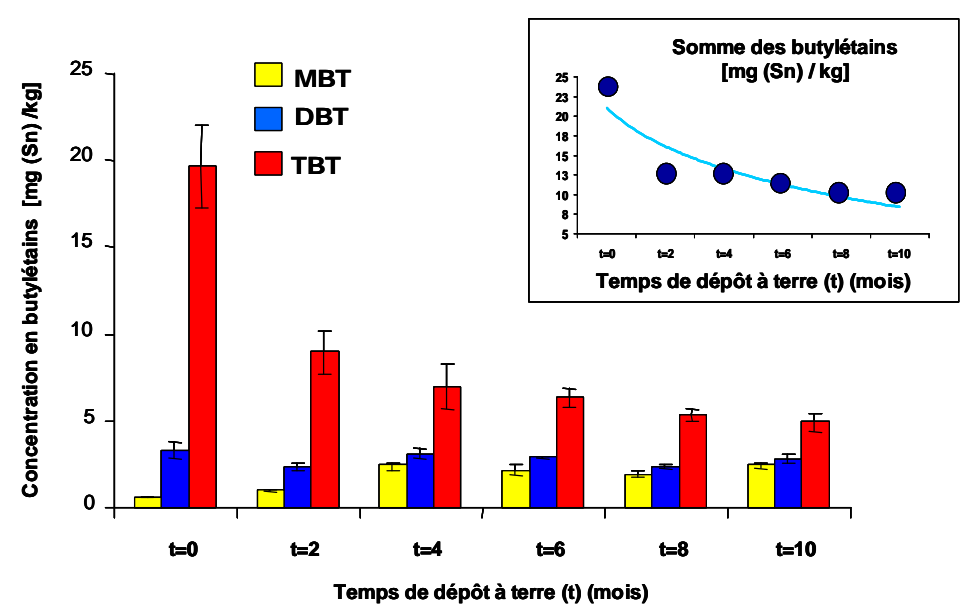

Figure 3. Evolution des butylétains dans les sédiments bruts avec le temps de dépôt à terre.

Indépendamment du type de sédiments, une diminution des concentrations en TBT est observée dans la figure 3. Ceci peut être expliqué par la dégradation naturelle du TBT en DBT, MBT puis en étain inorganique. Les quantités de DBT diminuent après 2 mois de stockage à terre et se stabilisent ensuite. Ceci est probablement dû à la production de DBT à partir de la dégradation du TBT. Les concentrations en MBT augmentent significativement pendant le dépôt à terre en raison de l'apport provenant de la dégradation du DBT.

Le profil du TBT en fonction du temps de dépôt à terre (figure 3) est très similaire à celui de la plupart des éléments traces avec une diminution importante des concentrations les deux premiers mois et une stabilisation ensuite. La dégradation du TBT est plus rapide pendant les 2 premiers mois avec une perte de la moitié de la concentration initiale. La cinétique de dégradation du TBT est ensuite plus lente. La rapide oxydation des sédiments dragués permet donc la dégradation du composé butylé le plus toxique. Après deux mois, la masse sédimentaire étant totalement oxydée, seule la dégradation microbiologique jouerait un rôle important dans le devenir du TBT.

La dégradation du TBT a déjà été mise en évidence dans les sols (HEROULT et al., 2008) et les sédiments (SARRADIN et al., 1995 ; TESSIER et al., 2007). Dans la plupart de ces études, il a été montré que la dégradation des composés organostanniques est induite à la fois par des procédés biotiques et abiotiques (température, irradiation lumineuse, présence de catalyseurs organiques ou inorganiques, conditions d'oxydation) mais la voie biologique est considérée comme étant la plus significative et la plus rapide. Il semble toutefois que la voie abiotique ne doit pas être négligée (TESSIER et al., 2007). La dégradation est clairement identifiée comme étant due à une perte successive d'un seul groupement butyle. Selon les études, les temps de demi-vie du TBT dans les sédiments sont courts (32 jours dans un écosystème modèle d'eaux douces (TESSIER et al., 2007) et de l'ordre de 80 jours dans un sol dopé (HEROULT et al., 
2008). Les temps de demi-vie du TBT ont également été calculés dans une carotte de sédiments marins en considérant la profondeur correspondant à une période de 14 années. Dans ces conditions plus anoxiques, le temps de demi-vie est de 2,1 années (SARRADIN et al., 1995). Ceci montre que la dégradation du TBT semble favorisée en conditions aérées et la dégradation de la moitié du TBT initial peut être obtenue en quelques mois.

3.3 Evolution des composés du mercure dans les sédiments en fonction du temps de stockage à terre

Le mercure étant un élément présent avec de fortes teneurs, le dosage des espèces du mercure peut être intéressant pour une meilleure évaluation de la toxicité des sédiments. Le mercure dans les sédiments dragués est probablement présent sous forme inorganique (e.g. sulfures) mais pourrait être transformé en formes méthylées par l'activité microbiologique qui augmente avec le temps de dépôt à terre des sédiments. Toutefois, les analyses par GC-ICP-MS ont montré des concentrations en méthylmercure inférieures à $1 \mu \mathrm{g} \mathrm{kg}^{-1}$. Il semble donc que le mercure reste sous forme inorganique pendant les 10 mois de stockage à terre.

\subsection{Evolution des composés arséniés dans les sédiments en fonction du temps de stockage à terre}

Bien que l'arsenic total ne soit pas influencé par la présence de sable ou la durée du stockage à terre, des changements dans la distribution de ses espèces peuvent se produire. Il est important de caractériser ces changements car la toxicité et la mobilité de As sont fortement liées à sa forme chimique.

Seules les espèces inorganiques de l'arsenic ont été détectées dans les extraits de sédiment. L'absence de formes méthylées comme le DMA ou le MMA montre la faible activité de méthylation des sédiments même après 10 mois de stockage à terre. Des changements dans la répartition des espèces inorganiques de As ont toutefois été observés. Les résultats sont donnés figure 4.

La figure 4 montre une diminution de la concentration en As(III) avec le vieillissement du sédiment et, pendant le même temps, $\mathrm{As}(\mathrm{V})$ augmente. Au niveau des résultats expérimentaux, il faut noter que si l'on calcule les rendements d'extraction en tenant compte de la somme des deux espèces d'arsenic par rapport à l'arsenic total, ils sont assez faibles au point initial (25\% à t=0 mois, sédiments bruts), ils augmentent jusqu'à $44 \%$ (sédiments bruts, $\mathrm{t}=2$ mois) et se stabilisent ensuite autour de $80 \%$. Il semble donc que dans les conditions réductrices des premiers mois de stockage à terre des sédiments, l'arsenic, en grande majorité sous la forme III est présent dans des phases porteuses relativement insolubles. Avec le vieillissement du sédiment, As(V) devient prépondérant, ce qui s'accompagne d'une augmentation des rendements d'extraction. Il semble donc que $\mathrm{As}(\mathrm{V})$ soit alors présent dans des phases porteuses solubles dans 
l'acide phosphorique $1 \mathrm{M}$ (réactif d'extraction) différentes de celles initialement présentes.

Dans les conditions redox initiales du sédiment dragué, As est probablement présent dans des minéraux contenant des sulfures de fer très stables dans des conditions réductrices (O’NEILL, 1995 ; BOSTICK et al., 2004). La phase d'oxydation de la masse sédimentaire libère cet arsenic qui s’oxyde pour donner As(V). Cette forme peut donner des minéraux solides stables par précipitation avec des cations divalents comme $\mathrm{Ca}, \mathrm{Fe}, \mathrm{Mn}, \mathrm{Ni}$ ou $\mathrm{Pb}$ (O’NEILL, 1995). As(V) peut aussi être retenu par adsorption sur des phases minérales solides comme les oxydes de Fe, $\mathrm{Al}$ ou Mn (XU et al., 1988). $\mathrm{As}(\mathrm{V})$ est généralement considéré comme mobile mais sa mobilité peut être affectée par de nombreux paramètres physico-chimiques.

3.5 Evolution du chrome hexavalent dans les sédiments en fonction du temps de stockage à terre

Les concentrations en $\mathrm{Cr}(\mathrm{VI})$ dans les sédiments stockés à terre pendant 10 mois sont présentées figure 5 .

Sédiment dessablé

Sédiment brut
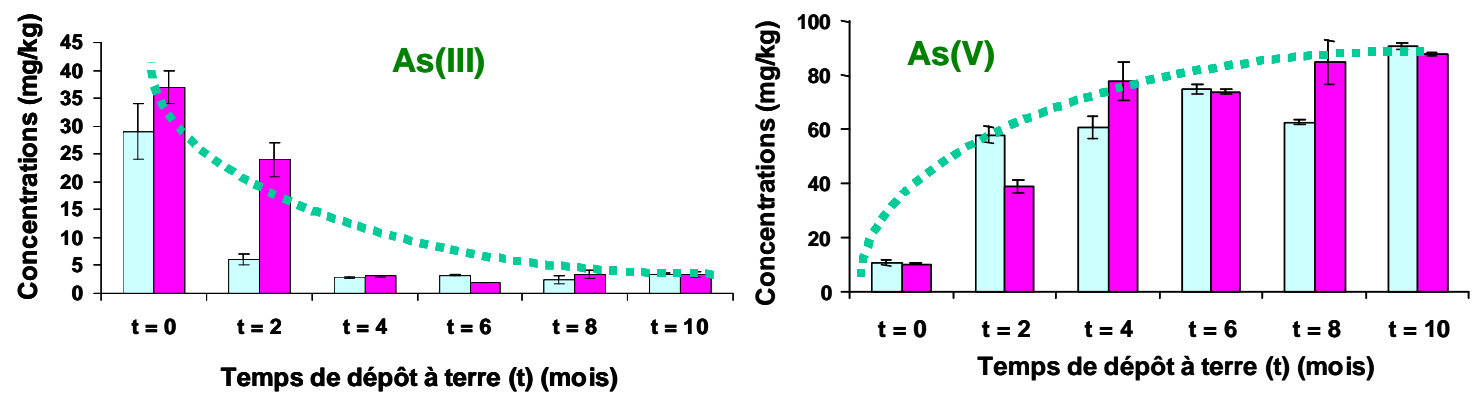

Figure 4. Evolution de As(III) et As(V) dans les sédiments en fonction du temps de stockage à terre.

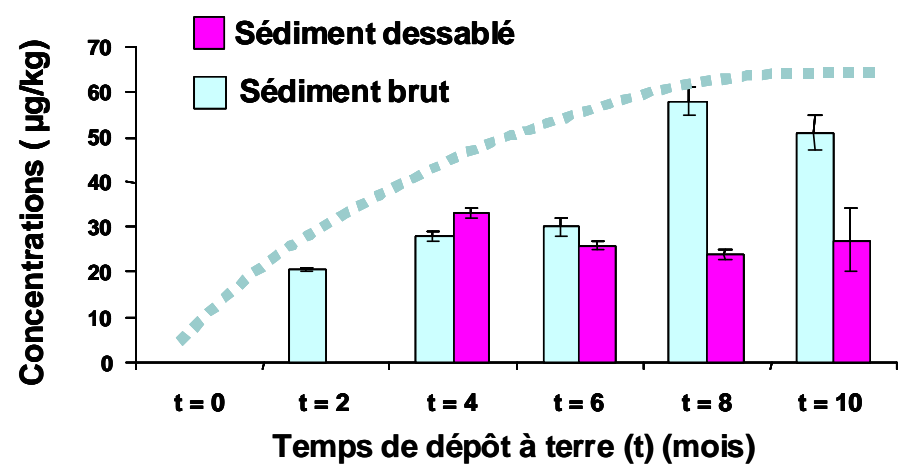

Figure 5. Concentration en Cr(VI) dans les sédiments marins dragués en fonction du temps de stockage à terre. 
Bien que le chrome hexavalent n’ait pas été détecté dans les sédiments fraîchement dragués, des traces de cette espèce sont présentes après 2 mois de stockage dans les sédiments bruts et après 4 mois dans les sédiments dessablés. Les très faibles teneurs expliquent la variabilité des résultats mais la formation de $\mathrm{Cr}(\mathrm{VI})$ semble se stabiliser après 4 mois. Ce comportement est très similaire à celui obtenu pour l'arsenic, ce qui confirme une oxydation globale du sédiment qui se produit dans les deux premiers mois après dragage. Initialement, le chrome est très probablement présent sous la forme trivalente mais s'oxyde lentement en $\operatorname{Cr}(\mathrm{VI})$. Il a été montré que le $\operatorname{Cr}(\mathrm{VI})$ n'est pas stable dans les sédiments anoxiques, particulièrement si la fraction de sulfures dites sulfures volatils acides est importante (RIFKIN et al., 2004 ; GRAHAM et al., 2009). Il est donc probable que cette fraction de sulfures disparaisse progressivement avec le stockage à terre des sédiments rendant possible l'oxydation d'une très faible partie du Cr(III). Plusieurs études ont mis en évidence que le $\mathrm{Cr}$ (III) des sédiments est essentiellement présent dans des phases minérales réfractaires et très faiblement mobilisables suggérant que l'oxydation du Cr(III) est minimale (GUO et al., 1997 ; GRAHAM et al., 2009). Ceci pourrait expliquer les faibles concentrations en Cr(VI) mesurées par rapport au chrome total de l'échantillon (proches de $100 \mathrm{mg} \mathrm{kg}^{-1}$ ). Ce faible taux d'oxydation diminue le risque environnemental associé à la présence de chrome dans les sédiments stockés à terre.

\section{Conclusion}

Ce travail, initié dans le cadre de SEDI.MAR.D 83, a été mené pour mieux comprendre le comportement des métaux et de leurs formes chimiques pendant le dépôt à terre de sédiments marins dragués pendant 10 mois. Les concentrations en élément total sont élevées et ne diminuent pas de façon importante pendant ce traitement. Des changements ont toutefois été observés au niveau des formes chimiques des éléments tels que $\mathrm{Sn}, \mathrm{Hg}$, As et Cr. Une diminution significative du tributylétain a été mise en évidence avec la durée du temps de stockage. La dégradation du TBT en des formes butylées qui sont moins dangereuses montre le grand intérêt du dépôt à terre pour diminuer la toxicité des sédiments dragués.

Aucune espèce méthylée du mercure et de l'arsenic n’a été mise en évidence pendant ce traitement montrant la faible activité de méthylation des sédiments étudiés. Seules les espèces inorganiques de l'arsenic sont présentes et un changement dans leur distribution a été observé avec le vieillissement. Les concentrations en As(III) diminuent alors que celles en $\mathrm{As}(\mathrm{V})$ augmentent. Cette oxydation a également été montrée pour le chrome avec la formation de $\mathrm{Cr}(\mathrm{VI})$ après 2 mois. Le Cr(VI) formé est cependant très bas par rapport aux teneurs en chrome total, ce qui ne semble pas présenter un risque environnemental important.

Ces résultats montrent l'intérêt du stockage à terre comme première approche peu coûteuse pour le traitement des sédiments marins dragués. Ce traitement nécessite 
Etude de l'évolution des formes chimiques des métaux dans des sédiments marins dragués stockés à terre : s3.11

toutefois un suivi chimique de qualité pour optimiser le temps de stockage à terre avant valorisation. Il serait maintenant intéressant de connaître l'évolution des composés organiques pour une gestion optimisée des sédiments en général.

\section{Références bibliographiques}

ALZIEU C. (2000). Environmental impact of TBT : The French experience, Science of the Total Environment, Vol. 258, pp 99-102.

AMIR S., HAFIDI M., MERLINA G., HAMDI H., REVEL J.C. (2005). Fate of polycyclic aromatic hydrocarbons during composting of lagooning sewage sludge, Chemosphere, Vol. 58, pp 449-458.

BOSTICK B.C., CHEN C., FENDORF S. (2004). Arsenic retention mechanisms within estuarine sediments of Pescadero, CA, Environmental Science \& Technology, Vol. 38, pp 3299-3304.

CAILLE N., TIFFREAU C., LEYVAL C., MOREL J.L. (2003). Solubility of metals in an anoxic sediment during prolonged aeration, Science of the Total Environment, Vol. 301, pp 239-250.

CAPPUYNS V., SWENNEN R., DEVIVIER A. (2006). Dredged river sediments : potential chemical time bombs ? a case study, Water, Air \& Soil Pollution, Vol. 171, pp 49-66.

CRAIG P.J. (1986) Organometallic compounds in the environment. Longman, London, $415 \mathrm{p}$.

DIRKX W.M.R., LOBINSKI R., ADAMS F.C. (1994). Speciation analysis of organotin in water and sediments by gas chromatography with optical spectrometric detection after extraction separation, Analytica Chimica Acta, Vol. 286, pp 309-318.

GRAHAM A.M., WADHAWAN A.R., BOUWER E.J. (2009). Chromium occurrence and speciation in Baltimore harbour sediments and porewater, Baltimore, Maryland, USA, Environmental Toxicology and Chemistry, Vol. 28, pp 471-480.

GUO T., DELAUNE R.D., PATRICK W.H. (1997). The influence of sediment redox chemistry on chemically active forms of arsenic, cadmium, chromium, and zinc in estuarine sediment, Environmental International, Vol. 23, pp 305-316.

HEROULT J., NIA Y., DENAIX L., BUENO M., LESPES G. (2008). Kinetic degradation processes of butyl- and phenyltins in soils, Chemosphere, Vol. 72, pp 940946.

KRETCHIK J.T. (2005). OSHA's direction, Chemical Health \& Safety, Vol. 12, pp 4444.

O’NEILL P. (1995). Arsenic. Dans: Heavy metals in soils, Blackie Academic \& Professional, London, pp 105-121. 
RODRIGUEZ MARTIN-DOIMEADIOS R.C., MONPERRUS M., KRUPP E., AMOUROUX D., DONARD O.F.X. (2003). Using speciated isotope dilution with GCinductively coupled plasma MS to determine and unravel the artificial formation of monomethylmercury in certified reference sediments, Analytical Chemistry, Vol. 75, pp 3202-3211.

RIFKIN E., GWINN P., BOUWER E. (2004). Chromium and sediment toxicity, Environmental Science \& Technology, Vol. 38, pp 267A-271A.

SARRADIN P.M., LAPAQUELLERIE Y., ASTRUC A., LATOUCHE C., ASTRUC M. (1995). Long term behaviour and degradation kinetics of tributyltin in a marina sediment, The Science of the Total Environment, Vol. 170, pp 59-70.

SEBY F., BENOIT-BONNEMASON C., DONARD O.F.X. (2007). Evolution des métaux et de leurs formes chimiques dans des vases portuaires dans le cadre d'un stockage à terre. Rapport de contrat SEDI.MAR.D. 83, 49 p.

SEBY F., GAGEAN M., GARRAUD H., CASTETBON A., DONARD O.F.X. (2003). Development of analytical procedures for determination of total chromium by quadrupole ICP-MS and high-resolution ICP-MS, and hexavalent chromium by HPLCICP-MS, in different materials used in the automotive industry, Analytical Bioanalytical Chemistry, Vol. 377, pp 685-694.

SINGH S.P., TACK F.M., VERLOO M.G. (1998). Heavy metals fractionation and extractability in dredged sediment derived surface soils, Water, Air and Soil Pollution, Vol. 102, pp 313-328.

STEPHENS S.R., ALLOWAY B.J., PARKER A., CARTER J.E., HODSON M.E. (2001). Changes in the leachability of metals from dredged canal sediments during drying and oxidation, Environmental Pollution, Vol. 114, pp 407-413.

TESSIER E., AMOUROUX D., MORIN A., LEHNOFF C., THYBAUD E., VINDIMIAN E., DONARD O.F.X. (2007). (Tri)Butyltin biotic degradation rates and pathways in different compartments of a freshwater model ecosystem, The Science of the Total Environment, Vol. 388, pp 214-233.

THOMAS P., FINNIE J.K., WILLIAMS J.G. (1997). Feasibility of identification and monitoring of arsenic species in soil and sediment samples by coupled highperformance liquid chromatography - Inductively coupled plasma mass spectrometry, Journal of Analytical Atomic Spectrometry, Vol. 12, pp 1367-1372.

VERMEULEN J., VAN GOOL M.P.M., MENTINK G.H., JOZIASSE J., BRUNING H., RULKENS W.H., GROTENHUIS J.T.C. (2007). Biochemical ripening of dredged sediments. Part 2. Degradation of polycyclic aromatic hydrocarbons and total petroleum hydrocarbons in slurried and consolidated sediments, Environmental Toxicology and Chemistry, Vol. 26, pp 2540-2549.

XU H., ALLARD B., GRIMVALL A. (1988). Influence of pH and organic substance on the adsorption of As(V) on geologic materials, Water, Air \& Soil Pollution, Vol. 40, pp 293-305. 